\title{
FISIOTERAPIA Y LA EDUCACIÓN A LOS PACIENTES \\ DE LA TÉCNICA INHALATORIA
}

Por: Esp. Andrés Flores Sancho

Recibido: 2019-03-28

Fisioterapeuta UCIMED

Publicado: 2019-04-29

(c) (1) $\odot$ (9)

Resumen:

as enfermedades del sistema respiratorio degeneran la salud en gran medida de la población mundial. El grupo de pacientes que se ve afectado por diferentes patologías neumológicas es vasto. Gracias a los avances tecnológicos y médicos muchas de estas enfermedades pueden ser controladas por diferentes métodos farmacológicos, pero la mala educación en cuanto a su correcto uso conlleva a una disminución del alcance positivo de estos fármacos. La razón de este artículo es promover la información con el fin de educar al personal en salud y a los pacientes sobre el correcto uso de los inhaladores como mecanismo farmacológico para mejor la calidad de vida.

\section{Abstract:}

The respiratory system diseases have an important global preponderance. The group of patients who are affected by different pneumological pathologies is vast. Thanks to technological and medical advances, many of them can be controlled by different pharmacological methods, but poor education regarding their correct use leads to a reduction in the positive reach of these drugs. The main reason for this article is to inform and educate health personnel and patients about the correct use of inhalers as a pharmacological mechanism for a better quality of life.

Palabras clave: enfermedad pulmonar, patología neumológica, EPOC, asma, dispositivos inhalatorios, técnica inhalatoria
Introducción

Las enfermedades del sistema pulmonar han sido de gran relevancia para la humanidad, ya que al ser uno de los sistemas más expuestos al medio ambiente lo convierte en un blanco fácil para microrganismos que coexisten en el entorno y buscan una oportunidad para atacar nuestro cuerpo. El sistema inmunológico, en conjunto con el respiratorio, están preparados para esto, de ahí las diferentes barreras para luchar contra estos microrganismos, ejemplos de estas barreras son los bellos nasales o mucosidades dentro del sistema.

Lamentablemente existen factores, que a pesar de tener estas barreras de protección, llegan a afectar nuestro organismo. Muestra de esto es la contaminación ambiental, el tabaquismo, el uso indebido de antibióticos y aunque cada vez menos, cocinar con leña, entre otros. Por esa razón, se han creado ciertas ayudas farmacológicas y físicas para mejorar la calidad de vida, disminuir la progresión patológica y así conseguir bajar la mortalidad por problemas agudos que afectan a pacientes de todas las edades.

La enfermedad pulmonar, una vista mundial Como se describe en el Foro de las Sociedades Respiratorias Internacionales (2017): "Mundialmente, al menos dos mil millones de personas están expuestas al humo tóxico del combustible de biomasa típicamente quemado de manera ineficiente en fogones de interiores mal ventilados. Mil millones de personas inhalan contaminantes atmosféricos al aire libre y otros 
tantos están expuestos al humo del tabaco. Aunque el deterioro respiratorio causa discapacidad y muerte en todas las regiones del mundo y en todas las clases sociales, la pobreza, el hacinamiento, las exposiciones ambientales $y$, en general, las malas condiciones de vida aumentan la vulnerabilidad a este grupo muy grande de trastornos"1.

Según los últimos estudios que se sometieron a revisión en este Foro Mundial, las cinco enfermedades respiratorias que más afectan a la población del planeta tierra son: enfermedad pulmonar obstructiva crónica (EPOC), asma, infecciones agudas, tuberculosis y cáncer que afecte algún órgano de este sistema. De estas cinco patologías, las dos con más impacto, EPOC y asma utilizan como medicamento diario los inhaladores. Este tipo de medicamento en muchas ocasiones se entrega por personal de la salud posterior a una cita con el neumólogo y ahí es donde puede existir un vacío educacional, ya que nadie prepara correctamente a este personal ni al paciente sobre una buena técnica inhalatoria.

Como lo publicaron en el periódico español La Provincia (2010): "El mal uso de los inhaladores resta eficacia a las terapias. Más de la mitad de los pacientes con enfermedad pulmonar y el $23 \%$ del personal sanitario no maneja de forma adecuada estos dispositivos indicados para el asma o el EPOC, el asunto no es insignificante si se tiene en cuenta que entre el 23 y el 85 por ciento (lo que significa una media del 50 por ciento) de los pacientes usuarios de estos dispositivos desconoce cómo usar los inhaladores de forma adecuada"2.

Este vacío en el uso correcto de estos dispositivos crea una progresión más rápida de la enfermedad, una disminución de la calidad de vida de estos pacientes y hasta en casos extremos agudos de asma podría llevar a la muerte.

\section{Técnica inhalatoria correcta}

El objetivo principal de este articulo además de evidenciar el vacío educacional que existe a nivel mundial y que muy probablemente puede ser traslapado a nuestro país, es dar un tutorial sobre la técnica inhalatoria correcta de los diferentes dispositivos que existen para las patologías pulmonares.

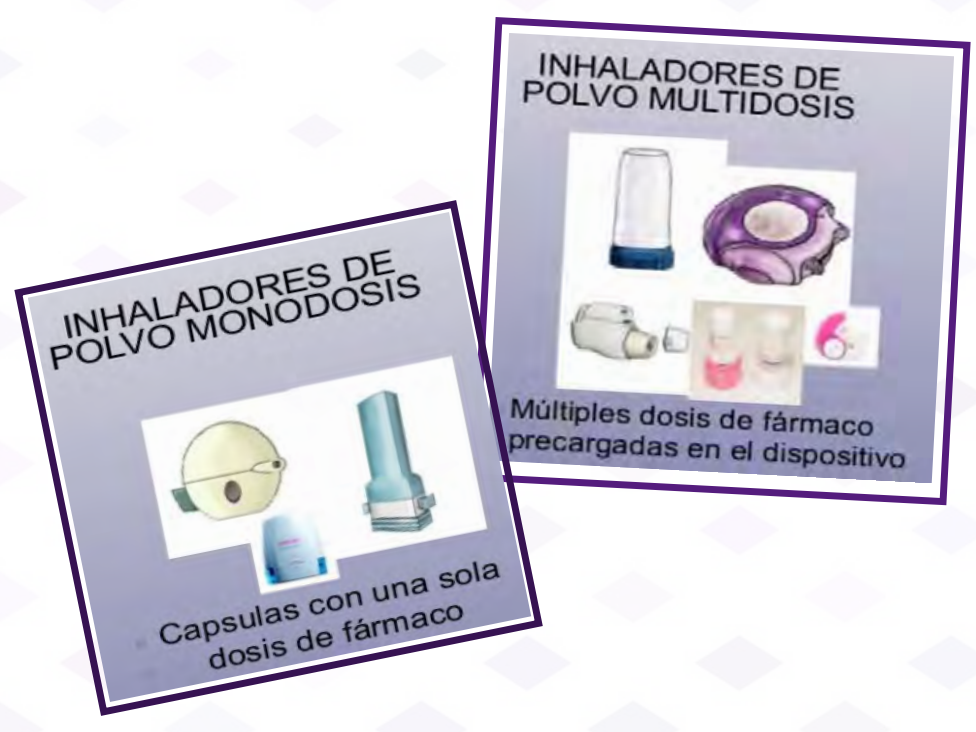

A pesar de la variedad de dispositivos inhalatorios, la técnica inhalatoria en todos es básicamente la misma, las variables son la forma de activar el medicamento que se va a utilizar, según del dispositivo. Puede ser polvo multidosis, en estos se activa mediante un puff o presión de un botón, lo cual expone la dosis para una inhalación dentro de la boca del paciente, estos dispositivos tienen un número específicos de puff, como común denominador 60 , pero cambia dependiendo la marca del mismo.

En el caso de los inhaladores monodosis, se debe preparar cada vez que se va a utilizar, introduciendo el medicamento en el dispositivo y activándolo. La mayoría de los pacientes tiene clara esta primera etapa del uso del medicamento. 


\section{Ahora se explica por pasos le técnica inhalatoria correcta.}

(1) Quitar la tapa del inhalador.

(2) Activar el inhalador (agitar enérgicamente de 5-6 veces) verticalmente.

(3) Exhalar la mayor cantidad de aire lenta y profundamente por la boca.

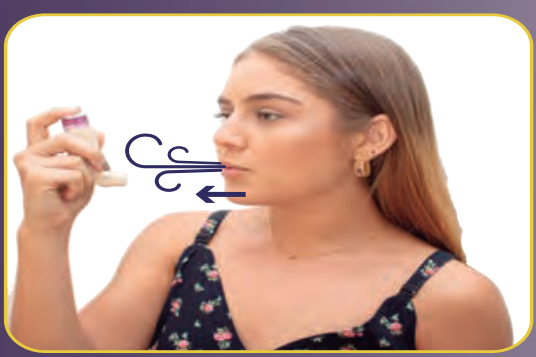

(4) Introducir el dispositivo y aprisionarlo entre los labios sin dejar que se escape el aire.

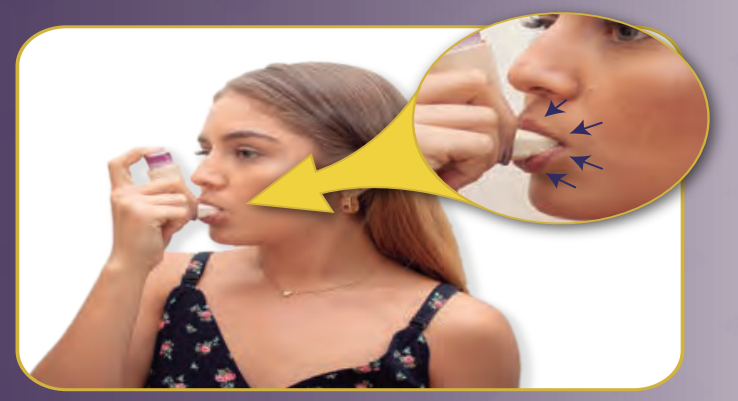

5 Este es el paso más difícil e importante, el paciente debe iniciar una inspiración lenta, coordinar presionar el cartucho, y hacer una inhalación profunda, donde sepa que el medicamento llegó hasta los pulmones, el lugar donde el fármaco realiza su trabajo.

(6) Sostener la respiración (apnea) por 10 segundos.

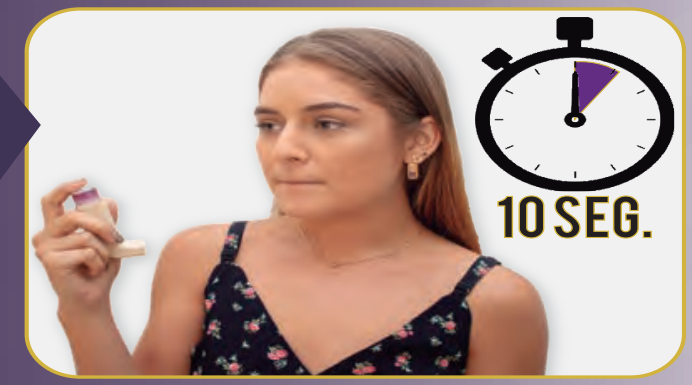

(7) Botar el aire lentamente por la nariz.

8 In caso de ser multidosis y si el paciente debe aplicar otro puff, esperar entre 30 y 60 segundos y volver a punto 2.

(9) Enjuagar la boca con agua para eliminar los residuos que algunos dispositivos dejan. 
A pesar de ser un protocolo bastante fácil y reproducible, gracias a diferentes estudios sabemos que los porcentajes de realizar una perfecta técnica inhalatoria a nivel pediátrico y adulto son bastante bajas. Por esta razón existe un dispositivo de ayuda para personas con problemas físicos o cognitivos para minimizar el desperdicio de medicamento. Este se usa con más frecuencia con niños $\mathrm{o}$ adultos mayores.

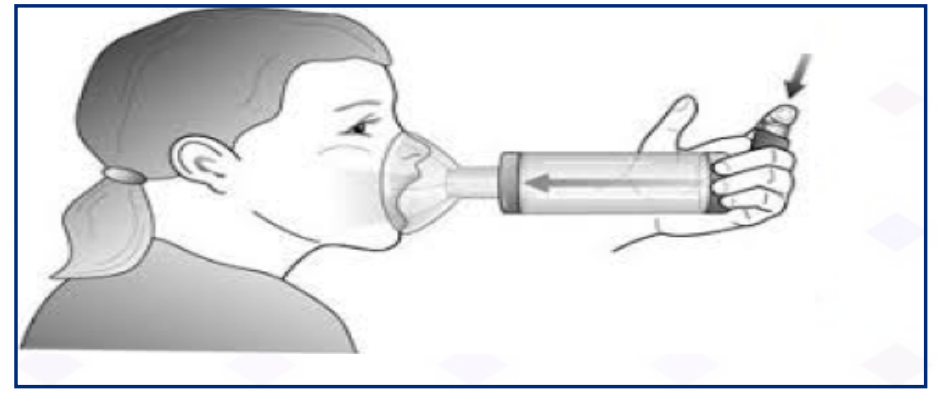

Espaciadores para dispositivos inhalatorios.

La Escuela de Kinesiología de la Universidad Santo Tomás, sede Viña del Mar, Chile en 2015, realiza una investigación en la cual los criterios de inclusión fueron, pacientes asmáticos, diagnosticados por medio de la prueba de función pulmonar, Espirometría. Las edades de los participantes estuvieron comprendidas entre 5 y 90 años, el número total de pacientes seleccionados fue de 270. La muestra final estuvo constituida por 135 pacientes pediátricos y 128 pacientes adultos.

Después de hacerles una revisión a los integrantes de la investigación, tomando en cuenta los pasos expuestos anteriormente, estos fueron los resultados de los errores más comunes de los pacientes al realizar la técnica inhalatoria.

\begin{tabular}{|c|c|c|c|c|}
\hline \multirow[t]{2}{*}{ Tipo de error } & \multicolumn{2}{|c|}{ Grupo pediátrico } & \multicolumn{2}{|c|}{ Grupo adulto } \\
\hline & $n^{2}$ & $\%{ }^{\circ}$ & $n^{4}$ & $\%^{\mathrm{b}}$ \\
\hline No exhalar antes de aplicar el inhalador & 5 & 3,7 & 68 & 53,1 \\
\hline No realizar apnea de 10 segundos & 11 & $8,1^{*}$ & 59 & $46,0^{\circ}$ \\
\hline No administrar sólo 1 puff a la vez & 4 & 3,0 & 37 & 28,0 \\
\hline No continuar inhalando después de activar el inhalador & 8 & 6,1 & 35 & 26,5 \\
\hline No activar el inhalador en la primera mitad de la inhalación & 4 & 3,0 & 30 & 22,7 \\
\hline No agitar el inhalador antes de usarlo & 0 & 0,0 & 25 & 18,9 \\
\hline No inhalar suave y profundamente mientras activa el inhalador & 4 & 3,0 & 14 & 10,6 \\
\hline No posicionar correctamente la aerocámara & 1 & 0,7 & 11 & 8,6 \\
\hline No sostener el inhalador vertical, con la boquilla hacia abajo durante el uso & 0 & 0,0 & 2 & 1,5 \\
\hline
\end{tabular}

Tabla 3. Frecuencia y porcentaje de errores en la técnica inhalatoria.

Esta tabla permite saber cuáles son los errores y dónde hay un vacío educacional más grande en los pacientes que tienen prescrito este tipo de medicamento. Algo importante de rescatar es que si se realiza un taller sobre la buena técnica inhalatoria, se debería hacer énfasis en los pasos que tienen más errores por parte de los pacientes. En esta investigación, el paso más errado fue la exhalación previa a la aplicación del inhalador, ya que se debe exhalar el mayor contenido gaseoso a nivel pulmonar, para que cuando se haga la inhalación del medicamento, la mayor cantidad del mismo pueda entrar a nuestros órganos respiratorios.

En segundo lugar, la apnea por 10 segundos, que significa mantenerse sin realizar inhalación o exhalación ninguna por 10 segundos, esto para lograr que el medicamento pueda realizar su trabajo dentro de las estructuras pulmonares.

Por otro lado, la investigación separó a los participantes por edad, para saber a cuál grupo etario se debería dar más reforzamiento educativo. Como se observa en la tabla 4 de la investigación.

\begin{tabular}{|c|c|c|}
\hline Edad (años) & $\begin{array}{l}\text { Ejecución } \\
\text { correcta }\end{array}$ & $\begin{array}{l}\text { Ejecución } \\
\text { incorrecta }\end{array}$ \\
\hline Pacientes pedtátricos & n & $\mathbf{n}$ \\
\hline $5-6$ & 5 & 3 \\
\hline $7-8$ & 12 & 1 \\
\hline $9-10$ & 14 & 7 \\
\hline $11-12$ & 19 & 11 \\
\hline 13-18 & 49 & 14 \\
\hline $\boldsymbol{x}$ & 73,4 & $26,6 *$ \\
\hline \multicolumn{3}{|l|}{ Pacientes adultos } \\
\hline $19-30$ & 9 & 3 \\
\hline $31-45$ & 0 & 6 \\
\hline $46-60$ & 1 & 24 \\
\hline $61-75$ & 3 & 48 \\
\hline $76-90$ & 1 & 35 \\
\hline x & 9,4 & $90,6 *$ \\
\hline
\end{tabular}

Tabla 4. Frecuencia de maniobras correctas/incorrectas por rango de edad ${ }^{4}$.

La investigación concluye que "La mayoría de los pacientes asmáticos pediátricos realiza la $\mathrm{TI}^{*}$ de manera correcta, mientras que aproximadamente el $90 \%$ de los pacientes adultos la ejecuta de manera incorrecta, siendo el error más común no exhalar antes de aplicar el inhalador. Se sugiere reforzar en los pacientes asmáticos, especialmente en los de mayor edad, la TI a través de nuevos métodos para lograr una correcta administración de sus medicamentos". ${ }^{4}$

Esta investigación representa en un alto porcentaje de problemas en el uso de estos dispositivos. Esto nos da una idea de qué es lo que podemos encontrar como personal de la salud cuando se realiza la revisión de la técnica inhalatoria a los pacientes, de ahí la importancia de este articulo y del reforzamiento constate que se debe dar a los pacientes que padecen estas patologías.

\section{${ }^{*} \mathrm{TI}=$ Técnica inhalatoria}

\section{BIBLIOGRAFÍA}

1.Muloiwa R. Impacto global de la enfermedad respiratoria. Foro de las sociedades respiratorias internacionales [Internet]. 2017; 2.

2.Sanatana C. El mal uso de los inhaladores resta eficacia a las terapias. La Provincia. 2010

3.Úbeda MC, Olga, al. E. Dispositivos de inhalación. Asociación española de pediatría en atención primaria. España: Grupo de Vías Respiratorias de la Asociación Española de Pediatría de Atención Primaria; 2013.

4.Manríquez P, Acuña AM, Muñoz L, Reyes A. Estudio sobre la técnica inhalatoria en pacientes asmáticos: diferencias entre pacientes pediátricos y adultos. JBP. 2015. 